\title{
Feedback control optimisation of ESR experiments
}

\author{
David L. Goodwin ${ }^{\mathrm{a}, \mathrm{b}}$, William K. Myers ${ }^{\mathrm{c}, *}$, Christiane R. Timmel ${ }^{\mathrm{c}}$, Ilya Kuprov ${ }^{\mathrm{a}, *}$ \\ ${ }^{a}$ School of Chemistry, University of Southampton, Highfield Campus, Southampton SO17 1 BJ, UK \\ ${ }^{b}$ Institute for Biological Interfaces 4 - Magnetic Resonance, Karlsruhe Institute of Technology (KIT), Fritz-Haber-Weg 6, 76131 Karlsruhe, Germany \\ ${ }^{c}$ Department of Chemistry, Centre for Advanced Electron Spin Resonance, University of Oxford, South Parks Road, Oxford OX1 3QR, UK
}

\begin{abstract}
Numerically optimised microwave pulses are used to increase excitation efficiency and modulation depth in electron spin resonance experiments performed on a spectrometer equipped with an arbitrary waveform generator. The optimisation procedure is samplespecific and reminiscent of the magnet shimming process used in the early days of nuclear magnetic resonance - an objective function (for example, echo integral in a spin echo experiment) is defined and optimised numerically as a function of the pulse waveform vector using noise-resilient gradient-free methods. We found that the resulting shaped microwave pulses achieve higher excitation bandwidth and better echo modulation depth than the pulse shapes used as the initial guess. Although the method is theoretically less sophisticated than simulation based quantum optimal control techniques, it has the advantage of being free of the linear response approximation; rapid electron spin relaxation also means that the optimisation takes only a few seconds. This makes the procedure fast, convenient, and easy to use. An important application of this method is at the final stage of the implementation of theoretically designed pulse shapes: compensation of pulse distortions introduced by the instrument. The performance is illustrated using spin echo and out-of-phase electron spin echo envelope modulation experiments. Interface code between Bruker SpinJet arbitrary waveform generator and Matlab is included in versions 2.2 and later of the Spinach library.
\end{abstract}

Keywords: ESR, AWG, feedback control, spin echo, OOP-ESEEM

PACS: 87.80.Lg, 02.60.Pn

\section{Introduction}

A significant current problem in high-field electron spin resonance (ESR) spectroscopy is the difficulty of achieving uniform and quantitative signal excitation using microwave pulses [1, 2]. The greatest instrumentally feasible electron spin nutation frequency in wide-band ESR spectrometers at W-band $(94 \mathrm{GHz})$ is about $50 \mathrm{MHz}$ [3]. The corresponding $\pi / 2$ pulse is therefore $5 \mathrm{~ns}$ long, and the excitation bandwidth is around $200 \mathrm{MHz}-$ enough to affect a significant portion of many solid state ESR signals, but insufficient to excite such signals uniformly and quantitatively. The consequences of partial excitation include useful orientation selection effects [4-6], but also reduced sensitivity and diminished modulation depth in two-electron dipolar spectroscopy [7-9].

The time resolution of the best available microwave pulse shaping equipment is of the order of $20 \mathrm{ps}^{1}$. This work uses Bruker SpinJet AWG with 0.625 ns time resolution - it enables generation of shaped pulses with the $(-3 \mathrm{~dB})$ bandwidth of about $330 \mathrm{MHz}$ and allows many broadband excitation schemes originally developed for nuclear magnetic resonance (NMR) spectroscopy [10] to be used with only minor modifications [11-15]. Numerically designed "optimal control" microwave

\footnotetext{
${ }^{*}$ Corresponding author

Email addresses: william.myers@chem.ox.ac.uk (William K.

Myers), i.kuprov@soton.ac.uk (Ilya Kuprov)

${ }^{1}$ Keysight M8196A 92 GSa/s Arbitrary Waveform Generator
}

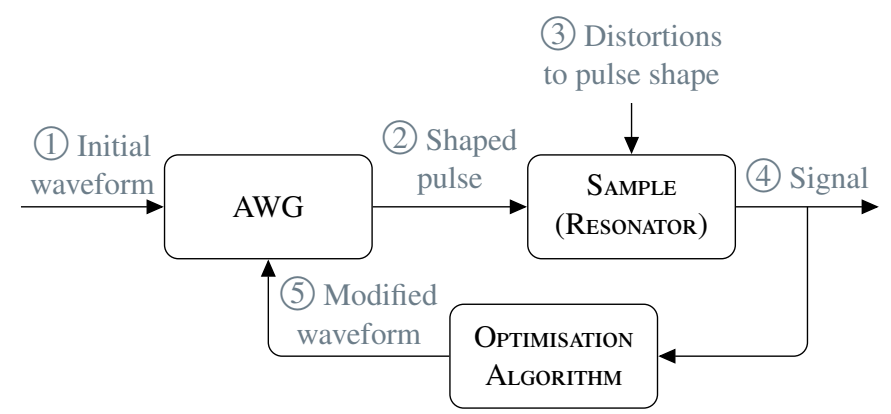

Figure 1: A schematic diagram of closed-loop feedback control: (1) Initial waveform sent to the AWG. (2) Waveform processed and sent to sample. (3) Distortions from noise and hardware response. (4) Sample excitation measured as a signal. (5) New pulse shape calculated and sent to the AWG.

pulses [16-19] are also possible [20, 21], but a complication specific to ESR is that the waveforms received by the sample are very different from those sent by the AWG - the distortions introduced by the ESR instrument cannot be ignored [20].

One way around this is to introduce a transfer matrix or a response function that connects, under the linear response approximation, the ideal pulse emitted by the computer to the real pulse seen by the sample $[22,23]$. The transfer matrix may be measured either by adding an antenna to the resonator [20], or by using a sample with a narrow ESR line to pick up the intensity of each frequency component [21]. Quasi-linear responses, such as phase variation across the excitation band- 
width, can be described with additional transfer matrices [12]. A common procedure is to apply the experimentally measured response function at the pulse optimisation stage, to send the result out of the AWG, and to hope that a good rendering of the intended pulse arrives at the sample point. It usually does $[20,21]$, but the downside is that the linear response assumption is hard-wired into the process. Measuring the response function with a sufficient signal-to-noise ratio can be time-consuming. ESR resonators, particularly at high frequencies, also tend to have strongly sample-dependent response functions.

In this communication, we explore a different microwave pulse shape refinement strategy that does not use the linear response approximation. It relies instead on the possibility of repeating an ESR experiment hundreds of times per second, and recognises the fact, discussed in detail below, that microwave pulse shapes in ESR need very few discretisation points.

The method is known as "feedback control" [24-26] and is illustrated schematically in FIG. 1. It was originally proposed in the context of MRI [27], NMR [28], and laser spectroscopy $[29,30]$. Its electron spin resonance adaptation is similar in its mathematical details to the well known (in the NMR circles) process of maximising the deuterium lock signal during the magnet shimming process [31] - a target variable is chosen and maximised, using a noise-resilient algorithm, with respect to the variables of interest. In relation to NMR pulse sequence optimisation, the method is known as "direct spectral optimisation" $[32,33]$.

In the ESR case explored in this work, the optimisation variables are either amplitudes of the microwave field at each time point, or parameters of the function describing the pulse shape. Improvements in excitation efficiency, spin-echo amplitude [34] and signal modulation depth in out-of-phase electron spin-echo envelope modulation (OOP-ESEEM) [35] experiments are demonstrated, at the instrument time cost not exceeding the time it used to take to auto-shim an NMR magnet minutes.

Although this method could be used to find an optimum pulse from a random guess, a more practical usage case would be to refine a pulse shape from some reasonably good starting point, such as a composite pulse or an open-loop optimal control solution [36], by compensating the inevitable distortions introduced by the ESR instrument $[37,38]$ in a way that does not rely on the linear response approximation.

\section{Feedback Control Optimisation}

\subsection{Arbitrary waveform generator interface}

The Bruker SpinJet AWG used in this work has a time resolution of $0.625 \mathrm{~ns}, 14$-bit amplitude resolution, $1.6 \mathrm{GS} / \mathrm{s} \mathrm{sam-}$ pling rate, and $\pm 400 \mathrm{MHz}$ range around the carrier frequency. The Bruker EleXSys II E580 ESR spectrometer has a 2 ns time base and, in combination with the AWG, resolves the time resolution mismatch by downsampling the pulse waveform onto a $1 \mathrm{~ns}$ increment time grid. The pulse response function up to the travelling wave tube (TWT) is shown in FIG. 2.

The software used in this work was written in-house, and has a flow of communication between Spinach [39] and Xepr

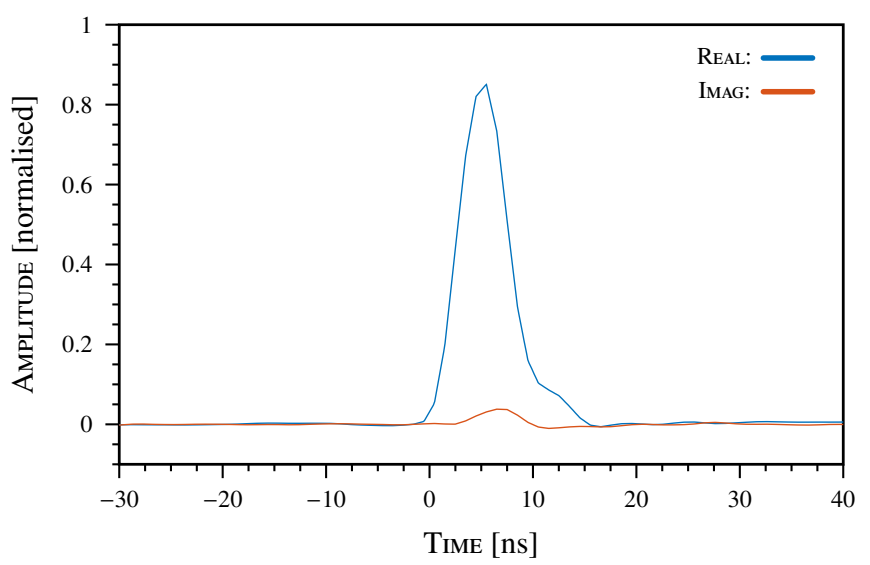

Figure 2: Pulse response function of our Bruker EleXSys II E580 spectrometer up to the TWT, measured by sending a large number of randomly generated waveforms (x) out of the AWG, recording the signals arriving at the transmitter monitor positioned just before the TWT $(\mathbf{y})$, and solving the overdetermined system of $\mathbf{y}=\mathbf{P x}$ equations for the transfer matrix $\mathbf{P}$ using the SVD pseudoinverse procedure. The plot shows one of the columns of $\mathbf{P}$.

Python libraries, shown in FIG. 3. The master process runs in Matlab and calls Xepr Python functions as necessary to control the instrument. Experimental data is written by Xepr into ASCII text files that are subsequently parsed by Matlab. Optimisation restart capability is implemented using an MD5 hash table of the previously submitted experimental settings and outcomes [18] - an interrupted optimisation can therefore retrace its steps quickly without re-running previously executed experiments.

To ensure that only feasible pulse shapes are sent from the software, the amplitude is folded into $[-1,+1]$ with the following mapping:

\begin{tabular}{|c|c|c|c|c|c|c|}
\hline \multicolumn{7}{|c|}{ Unconstrained Amplitude - Software Output } \\
\hline-3 & -2 & -1 & 0 & +1 & +2 & +3 \\
\hline $\mathrm{T}$ & $T$ & $T$ & $T$ & $T$ & $T$ & 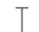 \\
\hline$\downarrow$ & $\downarrow$ & $\downarrow$ & $\downarrow$ & $\downarrow$ & $\downarrow$ & $\downarrow$ \\
\hline+1 & 0 & -1 & 0 & +1 & 0 & -1 \\
\hline
\end{tabular}

This is essentially a linear sawtooth map that takes $[-\infty,+\infty]$ into $[-1,+1]$ in a way that allows the optimisation algorithm to travel in $[-\infty,+\infty]$ and therefore removes the need to introduce constraints into the optimisation process.

\subsection{Optimisation method}

Numerical optimisation routines try to find extrema of the objective function supplied by the user [19, 40-42]. In ESR spectroscopy, the amplitude of a spin echo is a popular measure of sensitivity $[1,2]$ - the stronger the echo, as measured by the integral of its real part, the better the excitation efficiency. Formally, the echo intensity may be defined as a norm of the real part $s(t)$ of the echo signal [26], for example $L^{1}$-norm:

$$
\|s(t)\|:=\int_{0}^{T}|s(t)| \mathrm{d} t
$$




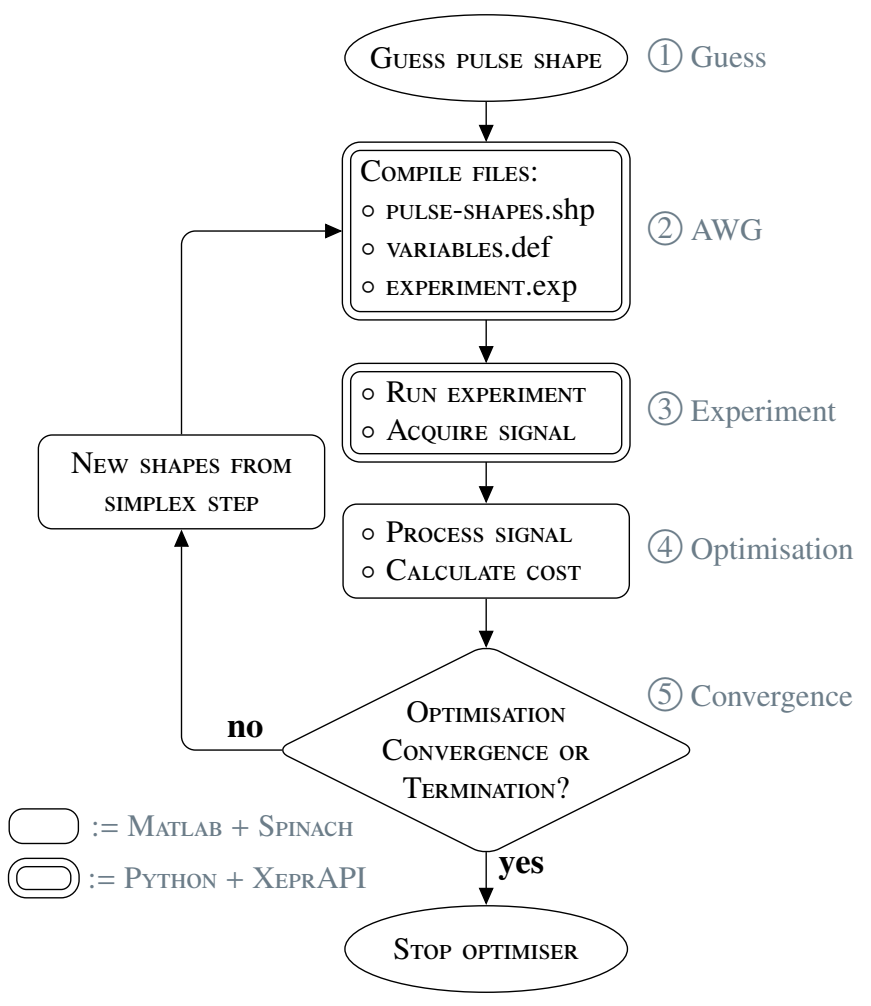

Figure 3: Software flow diagram: (1) Initial guess provided by user. (2) Waveform sent to AWG: load, show, and compile shape file, validate and compile PulseSPEL files. (3) Run experiment, read signal data. (4) Pass data to optimiser. (5) If no optimisation convergence, calculate new pulse shape.

where the integration is performed over the expected position of the echo (gained from an experiment with hard pulses). It is convenient to use a scaled objective function where the norm of the signal produced by the standard hard pulse experiment is used as a normalisation factor:

$$
Q[s(t)]=\frac{\|s(t)\|}{\|r(t)\|}
$$

Here, $Q$ is the functional to maximise, $s(t)$ is the real part of the signal measured after running an experiment, and $r(t)$ is the real part of the reference signal measured using hard pulses. $Q>1$ indicates a "better" echo than that from hard pulses alone, and $0<Q<1$ indicates a "worse" echo.

Numerical derivatives of noisy signals are unstable, and gradient-free optimisation strategies [19, 40-44] with a suitably scaled and bounded waveform are therefore to be preferred for feedback control optimisation. We found that a good choice is the Nelder-Mead algorithm [45, 46] - a member of the family of direct search methods known also as simplex methods [43], polytope methods [40] and ad hoc methods [41]. The method has the benefit of a smaller number of experimental evaluations per iteration compared to other gradient-free techniques, such as genetic algorithms [29, 47] or simulated annealing [48]. However, convergence is not guaranteed, and is linear at best [49]. A desirable benefit of the Nelder-Mead algorithm is its tolerance to random noise on a smooth function [50-52]. In practical testing, we have found that a particular modifica-

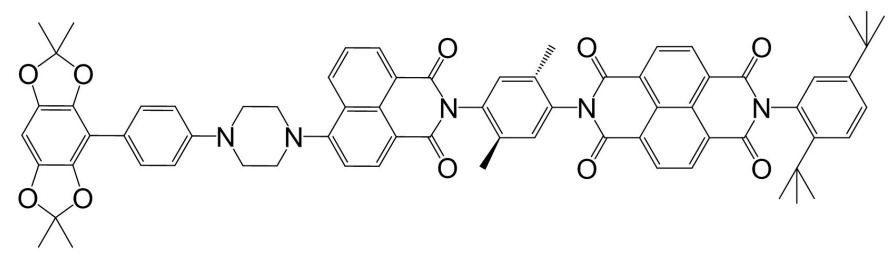

Figure 4: The sample used in OOP-ESEEM experiments on a photo-generated radical pair. The group that proposed this system [58] used BDX tag for the biphenyl dioxolane derivative on the left, ANI tag for the 4-aminonaphthalene1,8-imide (middle), and NI tag for the naphthalene-1,8-imide-4,5-imide (right), hence the overall BDXANINI tag for the construct.

tion of the Nelder-Mead algorithm, called the multidirectional search method [19, 53-55], works best. Its performance appears to be comparable to gradient descent algorithms; it is also designed to cope with multiple local minima [50]. A further useful property of the multidirectional search algorithm is that it has guaranteed convergence [56].

\section{Materials and Methods}

ESR measurements were performed on a Bruker Biospin EleXSys II E580 spectrometer with a SpinJet AWG based on an SPDevices SDR14 PCI board with a 0.625 ns time base. Samples were held at $85 \mathrm{~K}$ in an Oxford Instruments CF935O cryostat under a flow of cold nitrogen gas, controlled by an Oxford Instruments Mercury temperature controller. At X-band, the Bruker Biospin ER4118-MD5-W1 sapphire dielectric resonator with dimensions $5 \mathrm{~mm}$ ID, $10 \mathrm{~mm}$ OD and $13 \mathrm{~mm}$ height was used. The resonator was overcoupled for pulsed measurements to the quality factor of about 200.

For spin echo optimisation, the test sample was $2.0 \mu \mathrm{M}$ Finland trityl [57] dissolved in a mixture of $30 \%$ (by volume) glycerol-d $\mathrm{d}_{8}$ and $70 \% \mathrm{D}_{2} \mathrm{O}$.

OOP-ESEEM experiments used a sample of BDXANINI (FIG. 4), with the expected donor-acceptor distance of 26 $\AA$, similar to Sample 1 in [58], but with naphthalimide (NI) rather than phthalimide (PI) derivative as the acceptor, and 2,5ditertbutylphenyl as the end group. BDXANINI was dissolved in 4-cyano-4' -pentylbiphenyl (5CB) liquid crystal at a concentration of $0.2 \mathrm{mM}$ and degassed in a $4 \mathrm{~mm}$ OD quartz tube using the freeze-pump-thaw method prior to flame sealing under vacuum. Prior to measurements, the sample was heated up to the isotropic phase and frozen. Photoexcitation of the BDXANINI sample was performed with a Continuum Surelite Nd:YAG laser (7 ns pulses at $10 \mathrm{~Hz}$ at $1064 \mathrm{~nm}, 1 \mathrm{~mJ}$ per pulse, frequency tripled to $355 \mathrm{~nm}$ ) attenuated with a $\lambda / 2$ plate and depolarized. The beam was found to match the $5 \mathrm{~mm}$ cryostat window and was not manipulated further. Laser synchronisation was performed using a PatternJet II E580 board as an external trigger to a Stanford Research DG645 delay generator. 


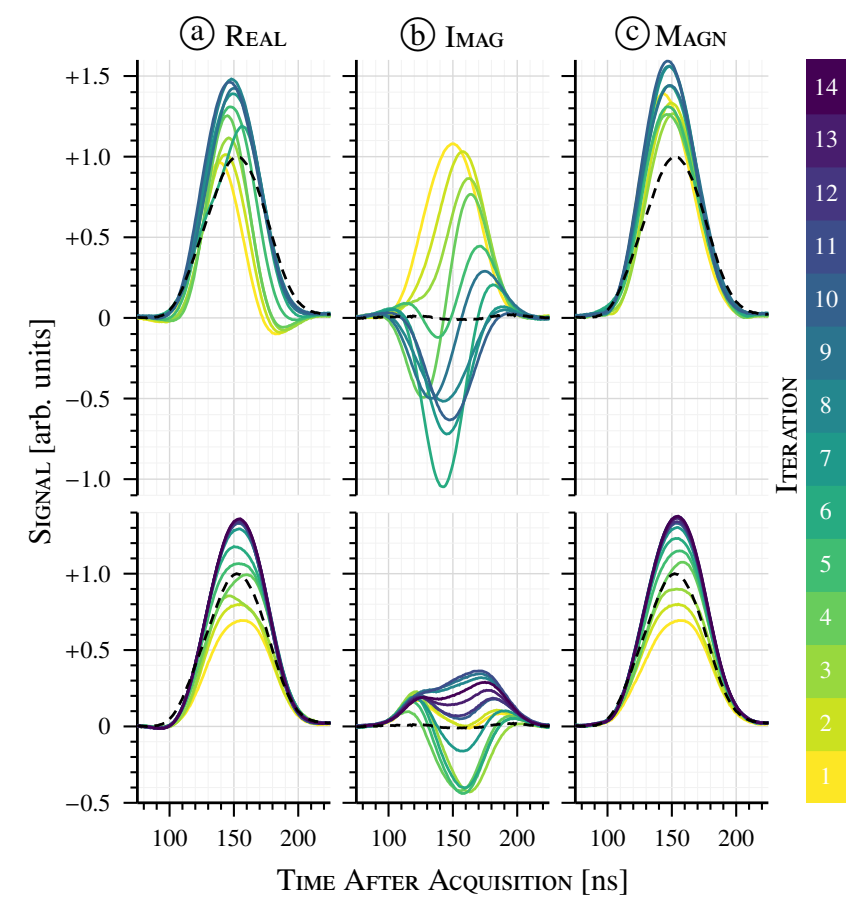

Figure 5: Optimisation process (colour-coded by iteration count) for a spin echo experiment performed on the trityl radical. The pulse waveform was optimised with respect to 11 discrete shape points (upper plots), and 21 discrete shape points (lower plots), with an overall $\pi$-pulse duration of $32 \mathrm{~ns}$. The resulting real (a), imaginary (b), and magnitude (c) components of the echo signal are shown. The hard pulse reference signal is indicated by a dashed line.

\section{Experimental Results}

\subsection{Feedback optimised spin echo}

A two-pulse spin echo experiment [34, 59], consisting of an excitation pulse followed by an inversion pulse,

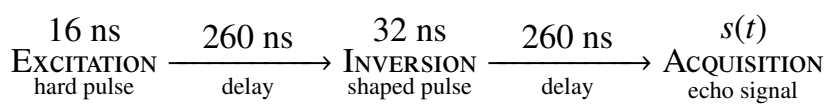

was used to test the performance of the feedback control optimisation. The $L^{1}$-norm of the real part of the echo was maximised by varying the shape (both the in-phase and the quadrature component) of a $32 \mathrm{~ns}$ inversion pulse with an echo delay of $260 \mathrm{~ns}$.

The initial condition was a random waveform. The echo signals at each iteration of the optimisation process are shown in FIG. 5 for the inversion pulse divided into $11(10 \times 3.2 \mathrm{~ns}$ time slices $)$ and $21(20 \times 1.6 \mathrm{~ns}$ time slices $)$ discrete points, interpolated linearly by the instrument. The Fourier transforms of these signals are shown in FIG. 6. Both pulses converge to an echo better than that of the optimum hard pulse from a variety of random initial guesses. Predictably, the 21-point shape takes more iterations to converge.

It is clear from FIG. 5 that a significant improvement in the echo intensity is accompanied by the appearance of out-ofphase components. This is the consequence of the target functional in EQ. 2 placing no constraints on the imaginary part of the echo signal. This is intentional for a simple demonstration: a different choice of the figure of merit (for example, a difference between the norm of the in-phase part and the norm of the

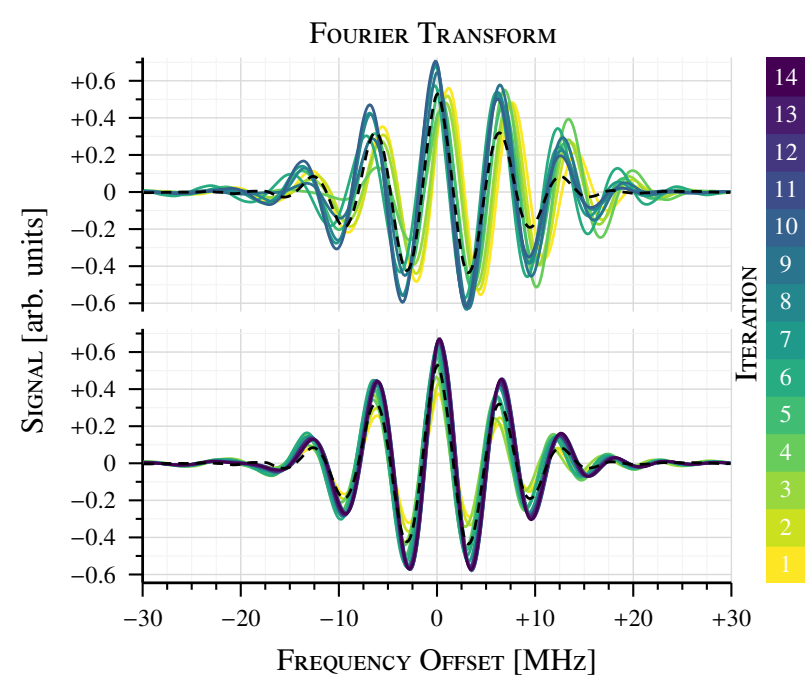

Figure 6: Optimisation process (colour-coded by iteration count) for a spin echo experiment performed on the trityl radical. The pulse waveform was optimised with respect to 11 discrete shape points (upper plots), and 21 discrete shape points (lower plots), with an overall $\pi$-pulse duration of $32 \mathrm{~ns}$. Fourier transforms (after zero-filling and a sine bell apodization) of the corresponding echo signals from FIG. 5 are shown. The hard pulse reference signal is indicated by a dashed line.

out-of-phase part) would suppress the out-of-phase component. A variety of other target functionals (for example, placing the magnetisation into a particular point on the Bloch sphere) and a discussion of their use may be found in the optimal control literature [16-19].

The pulse shapes at each iteration of the optimisation process are shown in FIG. 7. Although it is possible to run the optimisation with more waveform discretisation points, this was not in practice found to produce any further improvement. This may be rationalised by inspecting the spectrometer response function in FIG. 2, and a similar one published recently by the Prisner group [20]. The width of the kernel is around $5 \mathrm{~ns}$ even before the TWT and the resonator, and therefore all finer details of the pulse waveform are lost in the convolution process. It is then to be expected that the nearby points in a 21-point 32 ns waveform would become correlated, and this would make the job more difficult for the optimisation algorithm. Given the width of the response function, a more reasonable discretisation is about one point every $3 \mathrm{~ns}$ - almost exactly as in our 11-point shape shown in FIG. 5, where the optimiser performs well and runs in good time.

The convolution of the 11-point and the 21-point optimum pulses from FIG. 5 with the experimentally measured response function for our EleXSys II E580 spectrometer is shown in FIG. 7. An important conclusion is that the development of waveform shaping equipment in ESR spectroscopy should include improving the overall instrument response function there is little to gain from a sub-nanosecond AWG when the width of the response kernel, even without the amplifier or the resonator (FIG. 2), is several nanoseconds. 
(a) IN-PHASE - 11 POINT

(b) QuAdrature - 11 PoINT (C) In-Phase - 21 POINT

(d) QuADRATURE - 21 POINT

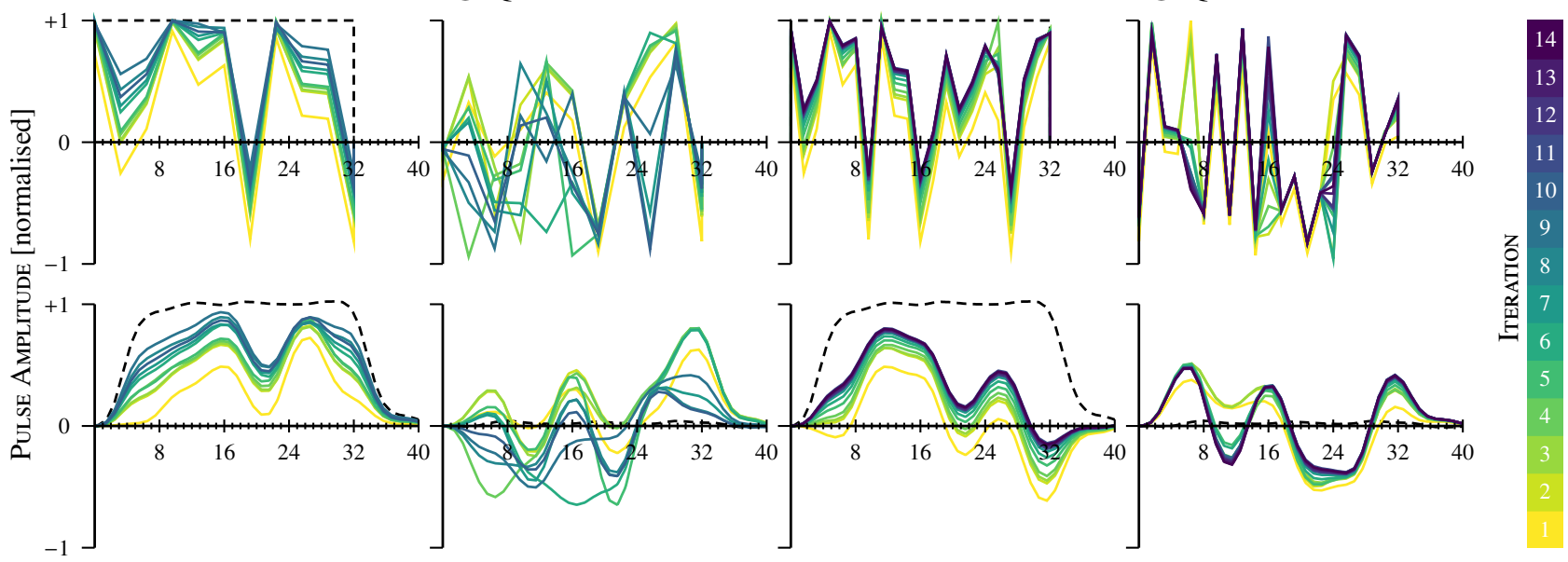

Time from Start of $\pi$-Pulse [ns]

Figure 7: Optimisation process (colour-coded by iteration count) for the in-phase and quadrature parts of (a-b) 11-point and (c-d) 21-point pulse shapes producing the echoes in FIG. 5. Lower plots show the convolution of the pulse shapes in the upper plots with the experimentally determined instrument response function. The hard pulse reference signal is indicated by a dashed line.

\subsection{Feedback optimised OOP-ESEEM}

A reasonable measure of sensitivity in an OOP-ESEEM experiment [35] is the modulation depth - the difference between the initial amplitude of the echo, and its amplitude at the first minimum [60, 61]. A norm of the difference between these echo signals is therefore one possible figure of merit:

$$
Q\left[s_{1}(t), s_{2}(t)\right]=\frac{\left\|s_{1}(t)-s_{2}(t)\right\|}{\left\|r_{1}(t)-r_{2}(t)\right\|}
$$

where $s_{1}(t)$ is the echo signal in an experiment with inter-pulse delay chosen to correspond to the first modulation maximum, $s_{2}(t)$ is the echo signal in an experiment with inter-pulse delay chosen to correspond to the first modulation minimum. Reference signals $r_{1}(t)$ and $r_{2}(t)$, produced by a sequence with hard pulses, are used for normalisation.

In this case, we chose to optimise the excitation pulse of the photo-induced OOP-ESEEM sequence:

$$
\underset{h v}{\text { LASER }} \rightarrow \underset{\text { shaped pulse }}{\text { EXCITATION }} \underset{\text { delay }+n \Delta}{\longrightarrow} \underset{\text { INVERSION }}{\text { hard pulse }} \underset{\text { delay }+n \Delta}{\longrightarrow} \text { ACQUISITION }
$$

The photogenerated BDXANINI biradical has the first modulation maximum at $16 \mathrm{~ns}$, and the first modulation minimum at 144 ns. These times were determined from an experiment with hard pulses, but it is also possible to allow these delays to vary during the optimisation.

The efficiency of the first pulse in ESEEM type sequences is a function of the bandwidth that the pulse is able to excite. Linear frequency sweep pulses [61], such as chirp [62, 63] and WURST (officially "wideband, uniform rate, smooth truncation" [64, 65], but more likely because the polar plot in 3D resembles a sausage), are successful for broadband excitation in ESR [11-13, 20, 61], and it is therefore reasonable to optimise the parameters of those pulses instead of treating the entire waveform as a variable vector.
The WURST pulse is defined by its amplitude and phase:

$$
A(t)=2 \pi \sqrt{\frac{\omega_{\mathrm{bw}}}{T}}\left(1-\left|\sin ^{a}\left(\frac{\pi t}{T}\right)\right|\right), \quad \varphi(t)=\pi \frac{\omega_{\mathrm{bw}}}{T} t^{2}+\varphi_{c}
$$

where $T$ is the pulse duration, $\omega_{\mathrm{bw}}$ is the excitation bandwidth, $a$ is the sine power used for the amplitude envelope, and $t$ runs over the interval $[-T / 2,+T / 2]$. The amplitude in EQ. 4 is normalised to produce a $\pi$-pulse. Other flip angles are obtained by changing the duration or the amplitude $[15,66]$.

Excitation pulse amplitude, duration, phase offset, and bandwidth were set as optimisation variables, and a phase cycle with $\varphi_{c} \in\{0, \pi / 2, \pi, 3 \pi / 2\}$ was set up. The initial guess was a $16 \mathrm{~ns}$ pulse with a zero phase offset, maximum amplitude, and a zero frequency sweep range, making it essentially a square pulse.

The small number of parameters makes the simplex method very efficient: FIG. 8 shows an improvement in the modulation depth by over $50 \%$ after just five iterations that ran in less than 3 minutes.

\subsection{Effect of instrument response function}

The excitation dynamics in the photo-generated BDXANINI biradical was simulated using Spinach library [39] for the corresponding two-electron spin system. Interaction parameters were those obtained by Wasielewski group [68] by fitting ESR data: the eigenvalues of $g$-tensors are $(2.0045,2.0045,2.0045)$ for the $\mathrm{BDX}^{+\bullet}$ donor and $(2.0032,2.0032,2.0011)$ for the $\mathrm{NI}^{-} \bullet$ acceptor. The exchange coupling is $180 \mu \mathrm{T}$ and the effective inter-electron distance is $26 \AA$.

The WURST pulse was applied numerically, and the fidelity calculated as the real part of the overlap between the final state of the system and the target state $|\sigma\rangle$ :

$$
\mathcal{F}=\operatorname{Re}\left\langle\sigma\left|\mathbf{P}_{N} \mathbf{P}_{N-1} \cdots \mathbf{P}_{2} \mathbf{P}_{1}\right| \rho_{0}\right\rangle
$$



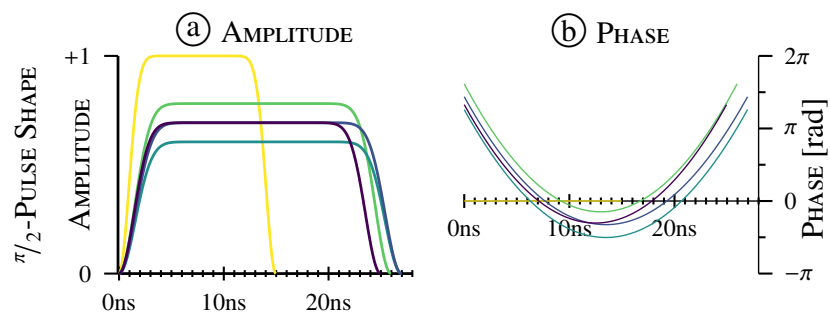

(c) REAL

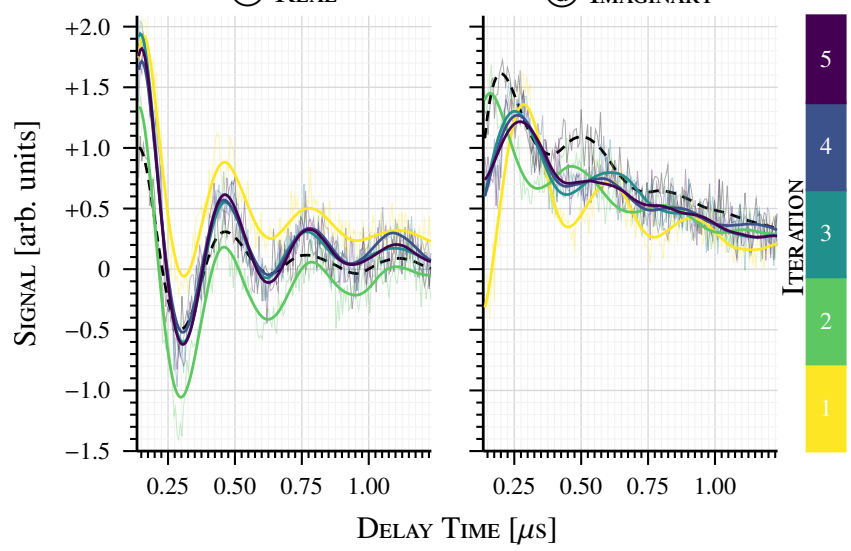

Figure 8: Modulation depth optimisation process (colour-coded by iteration count), showing (a-b) amplitude and phase of the excitation pulse, and (c-d) real and imaginary parts of the OOP-ESEEM signal. Thin lines show the raw data and bold lines are smoothed with a Savitzky-Golay filter [67] (25-point cubic $1^{\text {st }}$ pass, 75 -point quintic $2^{\text {nd }}$ pass). The hard pulse reference is indicated by a dashed line. The modulation depth is defined as the difference between the initial echo intensity and the intensity at the first minimum.

where the initial state $\left|\rho_{0}\right\rangle$ is the thermal equilibrium, and the propagator over an infinitesimally small time slice $\Delta t$ is

$$
\mathbf{P}_{n}=\exp \left(-i\left[\omega_{\mathrm{o}} \mathbf{H}_{\mathrm{Z}}+A_{n} \cos \left(\varphi_{n}\right) \mathbf{H}_{\mathrm{X}}+A_{n} \sin \left(\varphi_{n}\right) \mathbf{H}_{\mathrm{Y}}\right] \Delta t\right)
$$

where $\omega_{\mathrm{O}}$ is the resonance offset and $\mathbf{H}_{\mathrm{X}, \mathrm{Y}, \mathrm{Z}}$ are Cartesian spin operators. The destination state is in the XY plane with the phase matched to the offset in the following way:

$$
\begin{gathered}
|\sigma(\phi)\rangle=\sin (\phi)|x\rangle-\cos (\phi)|y\rangle \\
\phi\left(\omega_{\mathrm{o}}\right)=\frac{\pi T\left(\omega_{\mathrm{o}}-\omega_{\mathrm{o}}^{2}\right)}{\omega_{\mathrm{bw}}}
\end{gathered}
$$

The considerable effect of the instrument response function on the performance of the WURST pulse is illustrated in FIG. 9, where the left hand side shows excitation efficiency in the absence of any distortions, and the right hand side includes the effect of the response function. A reduction in the tolerance to the resonance offset is apparent.

Because the response function is a hard to predict collective property of the console, the resonator, and the sample, some degree of feedback optimisation is likely to be required in any experiment that seeks to attain maximum possible performance. A combination of analytical design and open-loop control, with feedback control as the last stage, is currently viewed as the most promising strategy [36].

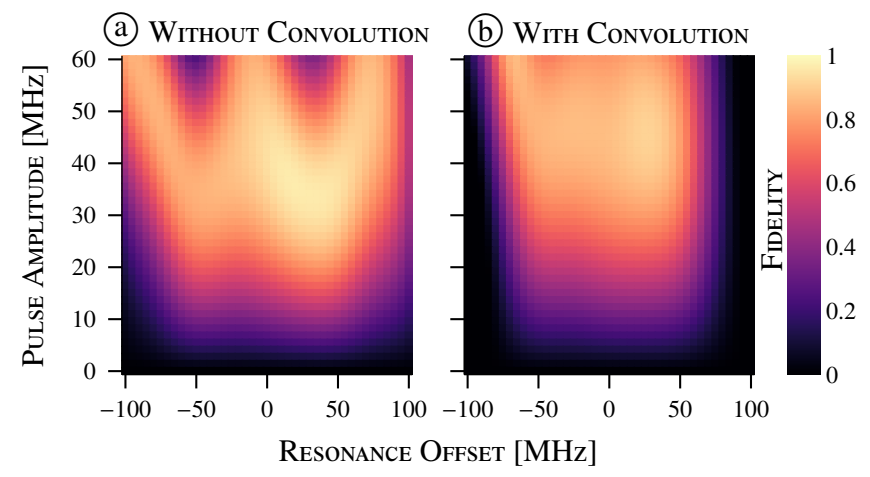

Figure 9: Robustness of the final pulse shape in FIG. 8 with respect to the instrument response convolution. Panel (a) shows the fidelity as a function of pulse amplitude and resonance offset for the theoretical WURST pulse shape with optimal parameters; panel (b) shows the fidelity achieved by a pulse that was convolved with the instrument response function shown in FIG. 7. The fidelity is normalised to the maximum achievable value.

\section{Discussion}

An important question regarding the improvements seen in the applications above is about the source of those improvements: what does a numerically optimised pulse do that a shaped pulse did not? This matter is discussed at length in the optimal control literature - the following factors are pertinent:

1. Because the optimum implies a zero gradient, optimised pulses are stable to first order with respect to variations in the optimised parameters, even in the presence of instrument distortions which may be significant (FIG. 7).

2. Feedback control optimisation does not make the linear response assumption that is implicit in any method that relies on measuring the response function. An experimentally measured response function may also be noisy and thus a limiting factor in the optimisation.

3. A typical frozen glass ESR sample contains distributions in a variety of parameters: spin system orientation, interaction amplitudes (for example, due to conformational mobility), $B_{1}$ field strength, temperature, etc. The pulse that comes out of a feedback optimisation does by definition have the best achievable performance, in the chosen class of functions, in the presence of all those distributions.

4. A numerically optimised pulse does not have to be adiabatic, and can explore the "shortcuts to adiabaticity" regime [69]. It is therefore possible to obtain a pulse reaching the target fidelity faster.

5. Feedback optimisation may be viewed as a generalisation of the pulse calibration process: more parameters than just duration and amplitude are now optimised, and pulse distortions by the instrument are hidden from view by the numerical optimisation algorithm.

The combination of these factors is responsible for the improved performance seen in FIGs. 5 and 8. Because so much of the ESR instrument response chain is sample-specific or hard to isolate, a feedback optimisation is nearly certain to result in 


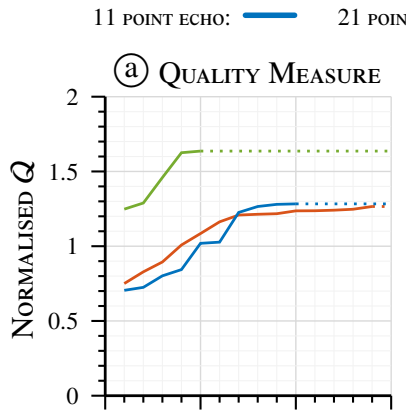

(C) Change in Quality

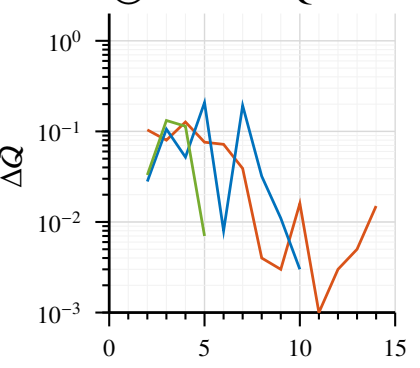

ITERATION

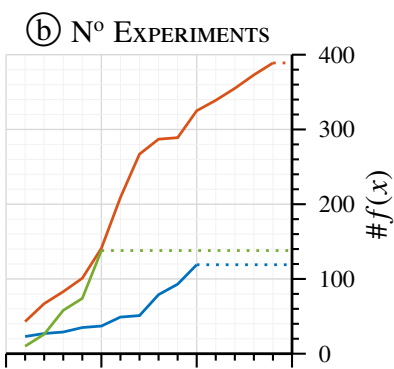

(d) Simplex Size

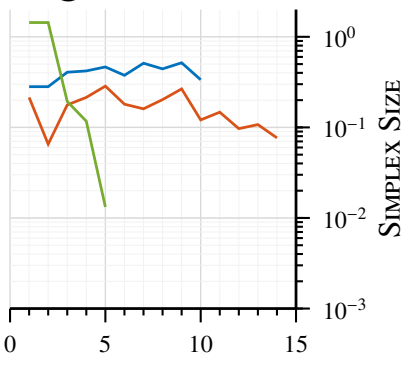

Figure 10: Convergence profiles for the pulse shape optimisations discussed in this work (echo pulse with 11 points, blue; echo pulse with 21 points, orange; OOP-ESEEM excitation pulse, green). The panels show (a) the quality metric as a function of the iteration count; (b) the cumulative number of ESR experiments performed by the instrument as a function of iteration count; (c) the change in the quality metric at each iteration; (d) the volume of the simplex at each iteration.

significant performance improvements with respect to the target metric specified by the user. In common with other applications of optimal control theory, the optimisations started from different random initial guesses show similar convergence characteristics and final achievable fidelity, but can converge to very different final pulse shapes. FIG. 10 shows an example of the optimisation convergence behaviour for spin echo and OOPESEEM optimisations.

The limitations of the feedback control method involve the following factors:

1. The initial signal-to-noise ratio. The $\mathrm{S} / \mathrm{N}$ produced by the initial guess must be sufficiently high for the optimisation to be able to start. Simplex optimisers are resilient to the presence of noise, but practical experience indicates that a signal-to-noise ratio of at least 5 is required for the optimisation to make progress from the starting point.

2. The choice of the fidelity measure. This is a caveat common to all optimal control theory [36]: the software will diligently maximise whatever it is given (for example, the real parts of the echoes in FIG. 5), potentially at the expense of other important factors (like the imaginary parts of the same echoes) if they are not included into the fidelity measure. Taking care in the formulation of the optimisation target is the users' responsibility.

3. Interpretability. This is traditionally the weak spot of optimal control theory, with several recent papers specifically dedicated to the ways of finding out what the optimal pulse actually does and why [70, 71]; this is difficult.

4. Transferability. Feedback optimised pulse shapes are not, in general, transferable between different samples or different experiments. However, an optimal solution from a different experiment or a previous sample is usually an excellent initial guess.

Optimisation targets used in this work (echo intensity and ESEEM modulation depth) are not the only ones possible. Almost any instrument output parameter may be declared a target, and an optimal pulse shape or shapes obtained that maximises this target. Nor is the approach restricted to pulses: delays are also valid optimisation coordinates, as are any other systematically variable instrument settings.

\section{Conclusions and outlook}

Simple feedback control methods using gradient-free algorithms to optimise the performance of shaped pulses can lead to significant signal amplitude and modulation depth improvements in ESR experiments. The principal advantage of feedback control is that the linear response approximation, and the consequent need to measure the instrument response function, are avoided entirely.

The method presented here should be seen as complimentary to other pulse shape optimisation techniques already used in ESR [11-15, 20, 21, 61, 66]. Feedback control optimisation is beneficial as a fine-tuning step in the application of existing pulse sequences and optimal control methods [37, 38] because it provides a simple and general way to take into account hardware-specific and sample-specific variations in the pulse shape distortions introduced by the ESR instrument.

Our interface code between Matlab and Bruker Xepr is available in versions 2.2 and later of the Spinach library [19, 39].

\section{Acknowledgements}

The authors are grateful to Arzhang Ardavan, Andrin Doll, Gunnar Jeschke, David Lurie, Burkhard Luy, and Thomas Prisner for insightful discussions, Michael Wasielewski for providing the sample material for OOP-ESEEM, and technical support of Bruker through Patrick Carl and Peter Höfer. This work was supported by the EPSRC (EP/L011972/1, EP/J500045/1), and EU FP7 (297861/QUAINT).

\section{References}

[1] A. Schweiger, G. Jeschke, Principles of pulse electron paramagnetic resonance, Oxford University Press, 2001.

[2] M. Brustolon, Electron paramagnetic resonance: a practitioner's toolkit, John Wiley \& Sons, 2009.

[3] P. A. S. Cruickshank, D. R. Bolton, D. A. Robertson, R. I. Hunter, R. J. Wylde, G. M. Smith, A kilowatt pulsed $94 \mathrm{GHz}$ electron paramagnetic resonance spectrometer with high concentration sensitivity, high instantaneous bandwidth, and low dead time, Rev. Sci. Instrum. 80 (10) (2009) 103102, doi:10.1063/1.3239402.

[4] Y. Polyhach, A. Godt, C. Bauer, G. Jeschke, Spin pair geometry revealed by high-field DEER in the presence of conformational distributions, J. Magn. Reson. 185 (1) (2007) 118-129, doi:10.1016/j.jmr.2006.11.012. 
[5] B. E. Bode, J. Plackmeyer, T. F. Prisner, O. Schiemann, PELDOR measurements on a nitroxide-labeled $\mathrm{Cu}$ (II) porphyrin: Orientation selection, spin-density distribution, and conformational flexibility, J. Phys. Chem. A 112 (23) (2008) 5064-5073, doi:10.1021/jp710504k.

[6] O. Schiemann, P. Cekan, D. Margraf, T. F. Prisner, S. T. Sigurdsson, Relative orientation of rigid nitroxides by PELDOR: Beyond distance measurements in nucleic acids, Angew. Chem. 48 (18) (2009) 3292-3295, doi:10.1002/anie.200805152.

[7] M. C. Thurnauer, J. R. Norris, An electron spin echo phase shift observed in photosynthetic algae: possible evidence for dynamic radical pair interactions, Chem. Phys. Lett. 76 (3) (1980) 557-561, doi:10.1016/00092614(80)80667-1.

[8] K. M. Salikhov, Y. E. Kandrashkin, A. K. Salikhov, Peculiarities of free induction and primary spin echo signals for spin-correlated radical pairs, Appl. Magn. Reson. 3 (1) (1992) 199-216, doi:10.1007/BF03166790

[9] J. Tan, M. C. Thurnauer, J. R. Norris, Electron spin echo envelope modulation due to exchange and dipolar interactions in a spin-correlated radical pair, Chem. Phys. Lett. 219 (3-4) (1994) 283-290, doi:10.1016/00092614(94)87059-4.

[10] S. Wimperis, Broadband, narrowband, and passband composite pulses for use in advanced NMR experiments, J. Magn. Reson. 109 (2) (1994) 221 231, doi:10.1006/jmra.1994.1159.

[11] P. E. Spindler, S. J. Glaser, T. E. Skinner, T. F. Prisner, Broadband inversion PELDOR spectroscopy with partially adiabatic shaped pulses, Angew. Chem. 52 (12) (2013) 3425-3429, doi:10.1002/anie.201207777.

[12] A. Doll, S. Pribitzer, R. Tschaggelar, G. Jeschke, Adiabatic and fast passage ultra-wideband inversion in pulsed EPR, J. Magn. Reson. 230 (2013) 27-39, doi:10.1016/j.jmr.2013.01.002.

[13] A. Doll, G. Jeschke, Fourier-transform electron spin resonance with bandwidth-compensated chirp pulses, J. Magn. Reson. 246 (2014) 18-26, doi:10.1016/j.jmr.2014.06.016.

[14] P. Schöps, P. E. Spindler, A. Marko, T. F. Prisner, Broadband spin echoes and broadband SIFTER in EPR, J. Magn. Reson. 250 (2015) 55-62, doi: 10.1016/j.jmr.2014.10.017.

[15] G. Jeschke, S. Pribitzer, A. Doll, Coherence transfer by passage pulses in electron paramagnetic resonance spectroscopy, J. Phys. Chem. B 119 (43) (2015) 13570-13582, doi:10.1021/acs.jpcb.5b02964.

[16] N. Khaneja, T. Reiss, C. Kehlet, T. Schulte-Herbrüggen, S. J. Glaser, Optimal control of coupled spin dynamics: design of NMR pulse sequences by gradient ascent algorithms, J. Magn. Reson. 172 (2) (2005) 296-305, doi:10.1016/j.jmr.2004.11.004.

[17] P. de Fouquieres, S. G. Schirmer, S. J. Glaser, I. Kuprov, Second order gradient ascent pulse engineering, J. Magn. Reson. 212 (2) (2011) 412 417, doi:10.1016/j.jmr.2011.07.023.

[18] D. L. Goodwin, I. Kuprov, Modified Newton-Raphson GRAPE methods for optimal control of spin systems, J. Chem. Phys. 144 (20) (2016) 204107, doi:10.1063/1.4949534.

[19] D. L. Goodwin, Advanced optimal control methods for spin systems, Ph.D. thesis, University of Southampton, UK, doi:10.5258/soton/t0003, 2017

[20] P. E. Spindler, Y. Zhang, B. Endeward, N. Gershernzon, T. E. Skinner, S. J. Glaser, T. F. Prisner, Shaped optimal control pulses for increased excitation bandwidth in EPR, J. Magn. Reson. 218 (2012) 49-58, doi: 10.1016/j.jmr.2012.02.013.

[21] T. Kaufmann, T. J. Keller, J. M. Franck, R. P. Barnes, S. J. Glaser, J. M. Martinis, S. Han, DAC-board based X-band EPR spectrometer with arbitrary waveform control, J. Magn. Reson. 235 (2013) 95-108, doi: 10.1016/j.jmr.2013.07.015.

[22] D. D. Rife, J. Vanderkooy, Transfer-function measurement with maximum-length sequences, J. Audio Eng. Soc. 37 (6) (1989) 419-444.

[23] P. E. Spindler, Electron paramagnetic resonance with shaped microwave pulses, Ph.D. thesis, Goethe Universität Frankfurt, 2014.

[24] G. F. Franklin, J. D. Powell, A. Emami-Naeini, J. D. Powell, Feedback control of dynamic systems, Addison-Wesley Reading, 1994.

[25] S. Skogestad, I. Postlethwaite, Multivariable feedback control: analysis and design, Wiley New York, 2007.

[26] J. C. Doyle, B. A. Francis, A. R. Tannenbaum, Feedback control theory, Courier Corporation, 2013.

[27] D. J. Lurie, A systematic design procedure for selective pulses in NMR imaging, Magn. Reson. Imag. 3 (3) (1985) 235-243, doi:10.1016/0730$725 \times(85) 90352-2$.
[28] H. Liu, S. J. Glaser, G. P. Drobny, Development and optimization of multipulse propagators: Applications to homonuclear spin decoupling in solids, J. Chem. Phys. 93 (11) (1990) 7543-7560, doi:10.1063/1.459386.

[29] R. S. Judson, H. Rabitz, Teaching lasers to control molecules, Phys. Rev. Lett. 68 (10) (1992) 1500, doi:10.1103/PhysRevLett.68.1500.

[30] C. J. Bardeen, V. V. Yakovlev, K. R. Wilson, S. D. Carpenter, P. M. Weber, W. S. Warren, Feedback quantum control of molecular electronic population transfer, Chem. Phys. Lett. 280 (1) (1997) 151-158, doi: 10.1016/S0009-2614(97)01081-6.

[31] A. E. Derome, Modern NMR techniques for chemistry research, Elsevier, 2013.

[32] G. De Paëpe, P. Hodgkinson, L. Emsley, Improved heteronuclear decoupling schemes for solid-state magic angle spinning NMR by direct spectral optimization, Chem. Phys. Lett. 376 (3) (2003) 259-267, doi: 10.1016/S0009-2614(03)00966-7.

[33] B. Elena, G. De Paëpe, L. Emsley, Direct spectral optimisation of protonproton homonuclear dipolar decoupling in solid-state NMR, Chem. Phys. Lett. 398 (4) (2004) 532-538, doi:10.1016/j.cplett.2004.09.122.

[34] E. L. Hahn, Spin echoes, Phys. Rev. 80 (4) (1950) 580, doi: 10.1103/PhysRev.80.580.

[35] C. R. Timmel, C. E. Fursman, A. J. Hoff, P. J. Hore, Spin-correlated radical pairs: microwave pulse effects on lifetimes, electron spin echo envelope modulations, and optimum conditions for detection by electron spin echo spectroscopy, Chem. Phys. 226 (3) (1998) 271-283, doi: 10.1016/s0301-0104(97)00283-8.

[36] S. J. Glaser, U. Boscain, T. Calarco, C. P. Koch, W. Köckenberger, R. Kosloff, I. Kuprov, B. Luy, S. Schirmer, T. Schulte-Herbrüggen, D. Sugny, F. K. Wilhelm, Training Schrödinger's cat: quantum optimal control, Eur. Phys. J. D 69 (12) (2015) 279, doi:10.1140/epjd/e201560464-1.

[37] M. Hellgren, E. Räsänen, E. K. U. Gross, Optimal control of strong-field ionization with time-dependent density-functional theory, Phys. Rev. A 88 (1) (2013) 013414, doi:10.1103/PhysRevA.88.013414.

[38] D. J. Egger, F. K. Wilhelm, Adaptive hybrid optimal quantum control for imprecisely characterized systems, Phys. Rev. Lett. 112 (24) (2014) 240503, doi:10.1103/PhysRevLett.112.240503.

[39] H. J. Hogben, M. Krzystyniak, G. T. P. Charnock, P. J. Hore, I. Kuprov, Spinach - A software library for simulation of spin dynamics in large spin systems, J. Magn. Reson. 208 (2) (2011) 179-194, doi: 10.1016/j.jmr.2010.11.008.

[40] P. E. Gill, W. Murray, M. H. Wright, Practical optimization, Academic Press, 1981

[41] R. Fletcher, Practical methods of optimization, Wiley, 2nd edn., 1987.

[42] J. Nocedal, S. J. Wright, Numerical optimization, Springer Science \& Business Media, 2006.

[43] W. H. Swann, Direct search methods, in: W. Murray (Ed.), Numerical Methods for Unconstrained Optimization, Academic Press, 13-28, 1972.

[44] R. P. Brent, Algorithms for minimization without derivatives, Courier Corporation, 2013.

[45] J. A. Nelder, R. Mead, A simplex method for function minimization, Comput. J. 7 (4) (1965) 308-313, doi:10.1093/comjnl/7.4.308.

[46] J. C. Lagarias, J. A. Reeds, M. H. Wright, P. E. Wright, Convergence properties of the Nelder-Mead simplex method in low dimensions, SIAM J. Optim. 9 (1998) 112-147, doi:10.1137/S1052623496303470.

[47] C. Brif, R. Chakrabarti, H. Rabitz, Control of quantum phenomena: past, present and future, New J. Phys. 12 (7) (2010) 075008, doi:10.1088/13672630/12/7/075008.

[48] N. Metropolis, A. W. Rosenbluth, M. N. Rosenbluth, A. H. Teller, E. Teller, Equation of state calculations by fast computing machines, J. Chem. Phys. 21 (6) (1953) 1087-1092, doi:10.1063/1.1699114.

[49] J. E. Dennis Jr, D. J. Woods, Optimization on microcomputers: The Nelder-Mead simplex algorithm, Tech. Rep., Rice University, Houston, TX, USA, doi:10.21236/ada453814, 1987.

[50] P. Gilmore, C. T. Kelley, An implicit filtering algorithm for optimization of functions with many local minima, SIAM J. Optim. 5 (2) (1995) 269285, doi:10.1137/0805015.

[51] D. M. Bortz, C. T. Kelley, The simplex gradient and noisy optimization problems, in: J. Borggaard, J. Burns, E. Cliff, S. Schreck (Eds.), Computational Methods for Optimal Design and Control, Springer, 77-90, doi: 10.1007/978-1-4612-1780-0_5, 1998.

[52] C. T. Kelley, Detection and remediation of stagnation in the Nelder-Mead 
algorithm using a sufficient decrease condition, SIAM J. Optim. 10 (1) (1999) 43-55, doi:10.1137/S1052623497315203.

[53] V. J. Torczon, Multi-directional search: a direct search algorithm for parallel machines, Ph.D. thesis, Rice University, Houston, TX, 1989.

[54] J. E. Dennis Jr,, V. Torczon, Direct search methods on parallel machines, SIAM J. Optim. 1 (4) (1991) 448-474, doi:10.1137/0801027.

[55] N. J. Higham, Optimization by direct search in matrix computations, SIAM J. Matrix Anal. Appl. 14 (2) (1993) 317-333, doi: $10.1137 / 0614023$.

[56] V. Torczon, On the convergence of the multidirectional search algorithm, SIAM J. Optim. 1 (1) (1991) 123-145, doi:10.1137/0801010.

[57] J. Ardenkjaer-Larsen, I. Laursen, I. Leunbach, G. Ehnholm, L.-G. Wistrand, J. Petersson, K. Golman, EPR and DNP properties of certain novel single electron contrast agents intended for oximetric imaging, Journal of Magnetic Resonance 133 (1) (1998) 1-12.

[58] R. Carmieli, Q. Mi, A. B. Ricks, E. M. Giacobbe, S. M. Mickley, M. R. Wasielewski, Direct measurement of photoinduced charge separation distances in donor-acceptor systems for artificial photosynthesis using OOP-ESEEM, J. Am. Chem. Soc. 131 (24) (2009) 8372-8373, doi: $10.1021 / \mathrm{ja} 902864 \mathrm{~h}$.

[59] L. G. Rowan, E. L. Hahn, W. B. Mims, Electron-spin-echo envelope modulation, Phys. Rev. 137 (1A) (1965) A61, doi:10.1103/PhysRev.137.A61.

[60] A. Doll, M. Qi, N. Wili, S. Pribitzer, A. Godt, G. Jeschke, Gd (III)-Gd (III) distance measurements with chirp pump pulses, J. Magn. Reson. 259 (2015) 153-162, doi:10.1016/j.jmr.2015.08.010.

[61] A. Doll, Frequency-swept microwave pulses for electron spin resonance, Ph.D. thesis, ETH Zürich, Switzerland, doi:10.3929/ethz-a-010670425, 2016.

[62] J. M. Böhlen, I. Burghardt, M. Rey, G. Bodenhausen, Frequencymodulated "Chirp" pulses for broadband inversion recovery in magnetic resonance, J. Magn. Reson. 90 (1) (1990) 183-191, doi:10.1016/00222364(90)90377-L.

[63] J. M. Böhlen, G. Bodenhausen, Experimental aspects of chirp NMR spectroscopy, J. Magn. Reson. A 102 (3) (1993) 293-301, doi: 10.1006/jmra.1993.1107.

[64] Ë. Kupče, R. Freeman, Adiabatic pulses for wideband inversion and broadband decoupling, J. Magn. Reson. A 115 (2) (1995) 273-276, doi: 10.1006/jmra.1995.1179.

[65] Ē. Kupče, R. Freeman, Stretched adiabatic pulses for broadband spin inversion, J. Magn. Reson. A 117 (2) (1995) 246-256, doi: 10.1006/jmra.1995.0750.

[66] P. E. Spindler, P. Schöps, W. Kallies, S. J. Glaser, T. F. Prisner, Perspectives of shaped pulses for EPR spectroscopy, J. Magn. Reson. 280 (2017) 30-45, doi:10.1016/j.jmr.2017.02.023.

[67] A. Savitzky, M. J. E. Golay, Smoothing and differentiation of data by simplified least squares procedures, Anal. Chem. 36 (8) (1964) 16271639, doi:10.1021/ac60214a047.

[68] M. T. Colvin, R. Carmieli, T. Miura, S. Richert, D. M. Gardner, A. L. Smeigh, S. M. Dyar, S. M. Conron, M. A. Ratner, M. R. Wasielewski, Electron spin polarization transfer from photogenerated spin-correlated radical pairs to a stable radical observer spin, J. Phys. Chem. A 117 (25) (2013) 5314-5325, doi:10.1021/jp4045012.

[69] E. Torrontegui, S. Ibáñez, S. Martínez-Garaot, M. Modugno, A. del Campo, D. Guéry-Odelin, A. Ruschhaupt, X. Chen, J. G. Muga, Chapter 2 - Shortcuts to Adiabaticity, in: E. Arimondo, P. R. Berman, C. C. Lin (Eds.), Advances in Atomic, Molecular, and Optical Physics, vol. 62, Academic Press, 117-169, doi:10.1016/B978-0-12-408090-4.00002-5, 2013.

[70] I. Kuprov, Spin system trajectory analysis under optimal control pulses, J. Magn. Reson. 233 (2013) 107-112, doi:10.1016/j.jmr.2013.02.012.

[71] S. Köcher, T. Heydenreich, S. Glaser, Visualization and analysis of modulated pulses in magnetic resonance by joint time-frequency representations, J. Magn. Reson. 249 (2014) 63-71, doi:10.1016/j.jmr.2014.10.004. 